\title{
LARGE MODELS OF COUNTABLE HEIGHT
}

\author{
BY
}

\section{HARVEY FRIEDMAN(1)}

ABSTRACT. Every countable transitive model $M$ of ZF (without choice) has an ordinal preserving extension satisfying $\mathrm{ZF}$, of power ${ }_{M \cap O n}$. An application to infinitary logic is given.

Any transitive model $M$ of ZFC with countably many ordinals must be countable. The situation is quite different when the axiom of choice is dropped.

The first examples of transitive models of ZF of power $\omega_{1}$ with countably many ordinals were constructed by Cohen. Later Easton, Solovay, and Sacks showed that every countable transitive model of $\mathrm{ZF}$ has an ordinal-preserving extension satisfying $Z F$, of power $2^{\omega}$. We prove here that every countable transitive model $M$ of $\mathrm{ZF}$ has an ordinal preserving extension satisfying $\mathrm{ZF}$, of power $\beth_{M \cap O n}$.

Theorem 1 is probably in the folklore. However, the proof of its first part is apparently not standard. The method used in that proof and the combinatorial construction of $\$ 2$ form the crux of the proof of the main theorem.

1. Adding subsets of $\omega^{\omega}$. Let $\omega=\{0,1,2, \cdots\}$, and identify $n$ with $\{0,1, \cdots, n-1\}$. Take $x^{<\omega}=\bigcup_{n} x^{n} . D \subset\left(\omega^{<\omega}\right)^{n}$ is dense if $\left(\forall x \in\left(\omega^{<\omega}\right)^{n}\right)$ $(\exists y \in D)(\forall i \in n)(x(i) \subset y(i)) . D \subset\left(\omega^{<\omega}\right)^{<\omega}$ is dense if $\left(\forall x \in\left(\omega^{<\omega}\right)^{<\omega}\right)$ $(\exists y \in D)(\forall i \in \operatorname{dom}(x))(x(i) \subset y(i))$.

Fix a countable transitive $M \vDash Z F$. An $x \in\left(\omega^{\omega}\right)^{n}$ is $M$-generic if for all dense $D \subset\left(\omega^{<\omega}\right)^{n}$ with $D \in M,(\exists y \in D)(\forall i \in n)(y(i) \subset x(i))$. An $x \in$ $\left(\omega^{\omega}\right)^{\omega}$ is M-generic if for all dense $D \subset\left(\omega^{<\omega}\right)^{<\omega}$ with $D \in M,(\exists y \in D)$ $(\forall i \in \operatorname{dom}(y))(y(i) \subset x(i))$. An $x \subset \omega^{\omega}$ is M-generic if any finite sequence of distinct elements of $x$ is $M$-generic, $x$ is infinite, and $\left(\forall y \in \omega^{<\omega}\right)(\exists z \in x)$ $(y \subset z)$. Let $M_{\alpha}$ be the sets in $M$ of rank $<\alpha$, for all $\alpha \in M$. For sets $x$, let $M_{\alpha}(x)$ be given by $M_{0}(x)=T C(\{x\}), M_{\alpha+1}(x)=\left\{y: y \in M_{\alpha}\right.$ or $y$ is first order definable over $\left(M_{\alpha}(x), \epsilon\right)$ with parameters allowed\}, $M_{\mu}(x)=$ $\bigcup_{\alpha<\mu} M_{\alpha}(x)$, for $\alpha, \mu \in M, \mu$ a limit. Take $M(x)=\bigcup_{\alpha \in M} M_{\alpha}(x)$.

Received by the editors December 4, 1973.

AMS (MOS) subject classifications (1970). Primary 02H10; Secondary 02 B25, $02 \mathrm{KO}$.

(1) This research was partially supported by NSF P038823. We wish to thank Ned Sturzer for correcting some errors that appeared in the original manuscript. 
LEMMA 1. If $x \subset \omega^{\omega}$ is M-generic and countable, then $x$ is the range of some M-generic $y \in\left(\omega^{\omega}\right)^{\omega}$.

Proof. Let $D_{0}, D_{1}, \cdots$ be an enumeration of all dense $D \subset(\omega<\omega)<\omega$ with $D \in M$. We wish to define an enumeration $x_{0}, x_{1}, \cdots$ of $x$ such that $\left(x_{0}, x_{1}, \cdots\right)$ is $M$-generic.

Let $y_{0}, y_{1}, \cdots$ be any fixed enumeration of $x$. For $j \in \omega$, we will define $x_{0}, \cdots, x_{i_{j}}$, where $i_{j}$ is a strictly increasing function of $j$. Take $i_{0}=0$, and $x_{0}=y_{0}$. Suppose $x_{0}, \cdots, x_{i_{j}}$ have been defined and are distinct elements of $x$. If $j$ is odd, set $i_{j+1}=i_{j}+1$, and $x_{i_{j+1}}$ to be the first element of $y_{0}, y_{1}, \cdots$ which has not appeared.

If $j$ is even, let $D=D_{j / 2}, k=i_{j}$. Let $E=\{r \mid k+1: r \in D$, $\operatorname{dom}(r)>k\}$. Then $E \subset\left(\omega^{<\omega}\right)^{k+1}$ is dense. By the $M$-genericity of $\left(x_{0}\right.$, $\left.\cdots, x_{k}\right)$, let $s \in E$ have $s(i) \subset x_{i}, i \leqslant k$. Take $t \in D$ with $s \subset t$, and let $t=\left(t_{0}, \cdots, t_{p}\right), k \leqslant p$. Extend $x_{0}, \cdots, x_{i_{j}}$ to $x_{0}, \cdots, x_{i_{j}}, \cdots, x_{p}$, so that $t_{i} \subset x_{i}, 0 \leqslant i \leqslant p$, and the $x_{i}$ are distinct elements of $x_{\text {. }}$

LEMmA 2. If $x \in\left(\omega^{\omega}\right)^{n}$ is M-generic, $y \in\left(\omega^{\omega}\right)^{n}, x(i), y(i)$ differ finitely, for $i<n$, then $y$ is M-generic.

Proof. This is well known, by symmetry.

LEMma 3. If $x \in\left(\omega^{\omega}\right)^{\omega}$ and for each $n, x \vee n$ is M-generic, then there is an M-generic $y \in\left(\omega^{\omega}\right)^{\omega}$ such that $(\forall n)(y(n)$ and $x(n)$ are finitely different $)$.

Proof. Let $D_{0}, D_{1}, \cdots$ be an enumeration of all dense $D \subset\left(\omega^{<\omega}\right)^{<\omega}$ with $D \in M$. Let $x=\left(x_{0}, x_{1}, \cdots\right)$. We wish to define a $y=\left(y_{0}, y_{1}, \cdots\right)$ which is $M$-generic, such that $x_{n}, y_{n}$ differ finitely.

For $j \in \omega$, we will define $y_{0}, \cdots, y_{i_{j}}$. Take $i_{0}=0, y_{0}=x_{0}$. Suppose $y_{0}, \cdots, y_{i_{j}}$ have been defined, and each $y_{i}, x_{i}$ are finitely different. Let $i_{j}=k$, and set $E \stackrel{j}{=}\left\{r \mid k+1: r \in D_{j}\right.$, dom $\left.(r)>k\right\}$. Then $\left.E \subset(\omega)^{<\omega}\right)^{k+1}$ is dense. By Lemma $2,\left(y_{0}, \cdots, y_{k}\right)$ is $M$-generic, and so let $s \in E$ have $s(i) \subset x_{i}$, $i \leqslant k$. Take $t \in D_{j}$ with $s \subset t$. Let $t=\left(t_{0}, \cdots, t_{p}\right), k \leqslant p$. Define $y_{k+1}$, $\cdots, y_{p}$ so that $t_{i} \subset y_{i}$ and $y_{i}$ differs finitely from $x_{i}$, for $k+1 \leqslant i \leqslant p$. Set $i_{j+1}=p$.

For $x \in \omega^{\omega}$, let $\bar{x}$ be the set of all $y \in \omega^{\omega}$ which are finitely different from $x$.

Let us call $\left(\bar{x}_{0}, \bar{x}_{1}, \cdots\right)$ M-generic if there is a sequence $\left(y_{0}, y_{1}, \cdots\right)$ with $y_{i} \in \bar{x}_{i}$, which is $M$-generic.

Lemma 4. $\left(\bar{x}_{0}, \bar{x}_{1}, \cdots\right)$ is M-generic if and only if, for each $n,\left(x_{0}, \cdots\right.$, $\left.x_{n}\right)$ is M-generic. 
Proof. If: Apply Lemma 3 to $\left(x_{0}, x_{1}, \cdots\right)=x$. Only if: By Lemma 2.

LEMMA 5. If $x \in\left(\omega^{\omega}\right)^{\omega}$ is M-generic, then $M(x) \vDash \mathrm{ZF}$.

Proof. This is well known.

LEMMA 6. If $M(x) \vDash \mathrm{ZF}, y \in M(x)$, then $M(y) \vDash \mathrm{ZF}$.

Proof. This is well known.

THEOREM 1. Let $M$ be a countable transitive model of ZF. If $y \subset \omega^{\omega}$ is $M$-generic then $M(y) \vDash \mathrm{ZF}$. If for each $n,\left(x_{0}, \cdots, x_{n}\right)$ is $M$-generic, then $M\left(\left(\bar{x}_{0}, \bar{x}_{1}, \cdots\right)\right) \vDash \mathrm{ZF}$.

Proof. Let $M$ be a countable transitive model of $\mathrm{ZF}, y \subset \omega^{\omega}$, where $y$ is $M$-generic. The question of whether $M(y) \vDash Z F$ is absolute. Hence if we can show that " $M(y) \vDash Z F$ " holds in some Boolean extension of the universe, we will have shown that $M(y) \vDash \mathrm{ZF}$ is in fact true.

We show that " $M(y) \vDash Z F$ " holds in any Boolean extension of the universe is which $y$ becomes countable. Argue as follows in the Boolean extension. By Lemma $1, y$ is the range for some $M$-generic $x \in\left(\omega^{\omega}\right)^{\omega}$. By Lemma 5 , $M(x) \vDash$ ZF. Since $y \in M(y)$, by Lemma 6 we have $M(y) \vDash$ ZF. We are done.

Now suppose that, for each $n,\left(x_{0}, \cdots, x_{n}\right)$ is $M$-generic. By Lemma 4, $\left(\bar{x}_{0}, \bar{x}_{1}, \cdots\right)$ is $M$-generic, and so let $x=\left(y_{0}, y_{1}, \cdots\right)$ be an $M$-generic sequence of representatives. By Lemma $5, M(x) \vDash Z$ ZF. Note that $\left(\bar{x}_{0}, \bar{x}_{1}, \cdots\right)$ $\in M(x)$. Hence by Lemma $6, M\left(\left(\bar{x}_{0}, \bar{x}_{1}, \cdots\right)\right) \vDash \mathrm{ZF}$. We are done.

Now fix $D_{m}^{n}$ such that, for each $n \geqslant 1, D_{m}^{n}$ enumerates all dense $D \subset$ $\left(\omega^{<\omega}\right)^{n}$ such that $D \in M$. An $x \in\left(\omega^{<\omega}\right)^{n}$ is m-M-generic just in case for all $p \leqslant m$, $\left(\exists y \in D_{p}^{m}\right)(\forall i)(y(i) \subset x(i))$. (Assume $m \geqslant 1$.)

Lemma 7. Let $s_{1}, \cdots, s_{k} \in \omega^{<\omega}, s_{i} \subset s_{j} \leftrightarrow i=j$. Let $m \geqslant 1$. Then there are $t_{1}, \cdots, t_{k} \in \omega^{<\omega}$ such that $s_{i} \subset t_{i}$, and every sequence of distinct elements of $\left\{t_{1}, \cdots, t_{k}\right\}$ is $m$-M-generic.

Proof. Left to the reader.

LEMMA 8. There is a perfect tree such that any finite sequence of distinct infinite paths is M-generic.

Proof. For each $j$ we will define a set $T_{j} \subset \omega^{k}$, for some $k$. For $j=0$, set $T_{0}=\{\langle\rangle\}$. Suppose $T_{j}$ has been defined, $T_{j} \subset \omega^{k}$. Suppose $j$ is odd. Set $T_{j+1} \subset \omega^{k+1}, T_{j+1}=\left\{s \cup\{\langle k, i\rangle\}:(i=0\right.$ or $\left.i=1) \& s \in T_{j}\right\}$.

Suppose $j$ is even, $T_{j} \subset \omega^{k}$. Let $j=2 m$. By Lemma 7, we can take $T_{j+1} \subset \omega^{q}$, some $q>k$, so that $\left(\forall s \in T_{j+1}\right)\left(\exists t \in T_{j}\right)(t \subset s),\left(\forall s \in T_{j}\right)$ 
$\left(\exists t \in T_{j+1}\right)(s \subset t)$, and every finite sequence of distinct elements of $T_{j+1}$ is $m+1-M$-generic.

Finally, let $T$ be the set of all $s \in \omega^{<\omega}$ such that for some $t \in \bigcup_{j} T_{j}$, we have $s \subset t$. It is easily verified that $T$ is a perfect tree. Let $x_{1}, \cdots, x_{k}$ be distinct infinite paths through $T$. Choose $j$ odd, $q \in \omega$ so that $T_{j} \subset \omega^{q}$, and the $x_{i}\left\lceil q\right.$ (which of course must be in $\left.T_{j}\right)$ are distinct. Then $\left(x_{1} \mid q\right.$, $\left.\cdots, x_{k} \mid q\right)$ is $m$ - $M$-generic, where $j=2 m+1$. Since $j$ may be chosen to be arbitrarily large, it is clear that for each $m$ there is a $q$ such that $\left(x_{1} \uparrow q\right.$, $\left.\cdots, x_{k}, q\right)$ is $m$-M-generic. Hence $\left(x_{1}, \cdots, x_{k}\right)$ is $M$-generic.

COROLlARY. If $M$ is a countable transitive model of $\mathrm{ZF}$, then there are M-generic $x \subset \omega^{\omega}$ of power $2^{\omega}$, and hence $x \subset \omega^{\omega}$ of power $2^{\omega}$ such that $M(x) \vDash \mathrm{ZF}$.

Proof. Immediate from Theorem 1 and Lemma 8.

2. A combinatorial lemma. For sets $x$, we say that $y \subset P(x)$ is independent just in case $\bigcap_{k=1}^{n} \pm y_{k}$ is infinite, where $n \geqslant 1, y_{1}, \cdots, y_{n}$ are distinct elements of $y$, and $+y_{k}=y_{k},-y_{k}=x-y_{k}$. In other words, any nontrivial Boolean combination of the elements of $y$ is infinite. Let $x \Delta y$ be $(x-y) \cup(y-x)$. Take $\bar{x}=\{y: x \Delta y$ is finite $\}$. For functions $f, g$ with domain an unbounded subset of $\lambda$, write $f \sim g$ for $(\exists \alpha<\lambda)(\forall \beta>\alpha)$ $(f(\beta)=g(\beta))$, and write $[f]=\{g: f \sim g\}$. Write $f / g$ for $(\exists \alpha<\lambda)(\forall \beta>\alpha)$ $(f(\beta) \neq g(\beta))$.

Let $a_{n}$ be of the set of multiples of the $n$th prime. It is clear that $\left\{a_{n}\right.$ : $0 \leqslant n\} \subset P(\omega)$ is independent. Let $f: \omega \rightarrow \omega$ be one-one onto.

By transfinite recursion, we define sets $A_{\alpha}^{f}, B_{\alpha}^{f}$ and functions $f_{\alpha}^{f}, g_{\alpha}^{f}$, for all ordinals $\alpha$, all one-one onto $f: \omega \rightarrow \omega$. Below it will be convenient to suppress the superscripts. Bear in mind that 0 is a nonlimit.

(1) $f_{0}=g_{0}=f, A_{0}=B_{0}=\omega$.

(2) $f_{\alpha+1}: \omega \rightarrow A_{\alpha+1}$ is given by $f_{\alpha+1}(n)=\left\{g_{\alpha}(k): k \in a_{n}\right\}$.

(3) $A_{\alpha+1}=\left\{y:(\exists n)\left(f_{\alpha+1}(n) \Delta y\right.\right.$ is finite $\left.)\right\}$.

(4) $B_{\alpha+1}=\left\{\bar{y}: y \in A_{\alpha+1}\right\}$.

(5) $g_{\alpha+1}: \omega \rightarrow B_{\alpha+1}$ is given by $g_{\alpha+1}(n)=\overline{f_{\alpha+1}(n)}$.

(6) $A_{\lambda}$ is the set of all functions $g$ whose domain is the nonlimits $\gamma<\lambda$, such that $g(\gamma) \in B_{\gamma}, g \mid \mu \in A_{\mu}$ for limits $\mu<\lambda$, and for some $n, g(\gamma)=$ $g_{\gamma}(n)$ for all sufficiently large $\gamma<\lambda$.

(7) $f_{\lambda}: \omega \rightarrow A_{\lambda}$ is given by $f_{\lambda}(n)(\gamma)=g_{\gamma}(n)$, for all nonlimits $\gamma<\lambda$.

(8) $B_{\lambda}=\left\{[h] \cap A_{\lambda}: h \in A_{\lambda}\right\}$.

(9) $g_{\lambda}: \omega \rightarrow B_{\lambda}$ is given by $g_{\lambda}(n)=\left[f_{\lambda}(n)\right] \cap A_{\lambda}$. 
We now let $C_{\alpha}, D_{\alpha}$, for $\alpha<\omega_{1}$, be any transfinite sequence of countable sets obeying

(a) $C_{0}=D_{0}=\omega$.

(b) $C_{\alpha+1}$ is the closure of some infinite independent subset of $P\left(D_{\alpha}\right)$ under finite symmetric difference.

(c) $D_{\alpha+1}=\left\{\bar{x}: x \in C_{\alpha+1}\right\}$.

(d) $C_{\lambda}$ is a set of functions $h$ with domain the nonlimits $\gamma<\lambda$ such that (i) $h(\gamma) \in D_{\gamma}$, (ii) $h \uparrow \mu \in C_{\mu}$, for limits $\mu<\lambda$, (iii) $\left(\forall g, h \in C_{\lambda}\right)$ ( $g \sim h$ or $g / h$ ), (iv) if $g \in C_{\lambda}, h$ has domain the nonlimits $\gamma<\lambda, h(\gamma) \in D_{\gamma}$, $h$ i $\mu \in C_{\mu}$ for limits $\mu<\lambda$, and $h \sim g$, then $h \in C_{\lambda}$, (v) $\left(\exists x \subset C_{\lambda}\right)(x$ infinite $\&(\forall g, h \in x)(g \neq h \rightarrow g / h))$.

(e) $D_{\lambda}=\left\{[f] \cap C_{\lambda}: f \in C_{\lambda}\right\}$.

We now fix $\delta<\omega_{1}$, and show that for some one-one onto $f: \omega \rightarrow \omega$, we have $A_{\alpha}^{f}=C_{\alpha}, B_{\alpha}^{f}=D_{\alpha}$, for all $\alpha<\delta$. It is convenient to assume that $\delta$ is a limit.

Let us call a class $K$ of functions $f_{\alpha}^{\prime}, g_{\alpha}^{\prime}, \alpha<\delta$, special just in case there is a $k$ such that

I. Each $f_{\alpha}^{\prime}, g_{\alpha}^{\prime}$ is a one-one finite partial map from $\omega$ into $C_{\alpha}, D_{\alpha}$ respectively.

II. $\left\{\alpha: f_{\alpha}^{\prime} \neq \varnothing\right\}$ contains only finitely many nonlimits.

III. $f_{\alpha}^{\prime}(n), g_{\alpha}^{\prime}(n)$ are undefined if $n>k$.

IV. For each $n,\left\{\alpha: g_{\alpha}^{\prime}(n)\right.$ is defined $\}$ is either finite or the union of a finite set with $\{\alpha: \alpha \leqslant \lambda\}$, for some limit $\lambda<\delta$.

V. If $f_{\alpha}^{\prime}(n)$ is defined, then $f_{\alpha}^{\prime}(n) \in g_{\alpha}^{\prime}(n) \in D_{\alpha}$, for $\alpha \neq 0$.

VI. $f_{0}^{\prime}=g_{0}^{\prime}$.

VII. $f_{\lambda}^{\prime}(n)$ is defined if and only if $g_{\alpha}^{\prime}(n)$ is defined for all $\alpha \leqslant \lambda$. If $f_{\lambda}^{\prime}(n)$ is defined, then $f_{\lambda}^{\prime}(n)(\gamma)=g_{\gamma}^{\prime}(n)$, for all nonlimits $\gamma<\lambda$.

VIII. Suppose $f_{\alpha+1}^{\prime}(n)$ is defined. If $m \in a_{n}, g_{\alpha}^{\prime}(m)$ is defined, then $g_{\alpha}^{\prime}(m) \in f_{\alpha+1}^{\prime}(n)$. If $m \notin a_{n}, g_{\alpha}^{\prime}(m)$ is defined, then $g_{\alpha}^{\prime}(m) \notin f_{\alpha+1}^{\prime}(n)$.

XI. If $f_{\alpha+1}^{\prime} \neq \varnothing$, then $g_{\alpha}^{\prime}(m)$ is defined for all $m \leqslant k$.

For classes $K, K^{*}$ of partial maps $f_{\alpha}^{\prime}, g_{\alpha}^{\prime}, \alpha<\delta, K^{*}$ extends $K$ if every $f_{\alpha}^{\prime}$ or $g_{\alpha}^{\prime}$ of $K$ is contained in the corresponding $f_{\alpha}^{\prime}$ or $g_{\alpha}^{\prime}$ of $K^{*}$. We also let " $K+f_{\alpha}(n)=y$ ", or " $K+g_{\alpha}(n)=y$ ", for $n \in \omega, \alpha<\delta$, be the extension of $K$ obtained by just extending the domain of $f_{\alpha}$, or $g_{\alpha}$, as indicated in the expression.

Call $K$ weakly special just in case there is a $k$ such that I-VIII hold.

Lemma 1. Let $K$ be weakly special, $m \in \omega, 0<\alpha<\delta$. Then for some $y$, $K+g_{\alpha}(m)=y$ is weakly special. If $\alpha=0$, then for some $y,\left(K+g_{\alpha}(m)=y\right)$ $+f_{\alpha}(m)=y$ is weakly special. 
Proof. Assume $g_{\alpha}^{\prime}(m)$ is undefined in $K$. Let $r_{1}, \cdots, r_{p}$ be a one-one enumeration of the arguments $r$ at which $f_{\alpha+1}^{\prime}$ is defined in $K$, with $m \in a_{r}$; let $s_{1}, \cdots, s_{q}$ be a one-one enumeration of the arguments $s$ at which $f_{\alpha+1}^{\prime}$ is defined in $K$, with $m \notin a_{s}$. Take

$$
x=\left(\bigcap_{i} f_{\alpha+1}^{\prime}\left(r_{i}\right)\right) \cap\left(\bigcap_{i}\left(D_{\alpha}-f_{\alpha+1}^{\prime}\left(s_{j}\right)\right)\right) .
$$

By I, clearly $x$ is infinite. Let $y \in x$, where $y$ is not in the range of $g_{\alpha}^{\prime}$ in $K$. Clearly $K+g_{\alpha}^{\prime}(m)=y$ is weakly special if $\alpha \neq 0$, and $\left(K+g_{\alpha}^{\prime}(m)=y\right)$ $+f_{\alpha}^{\prime}(m)=y$ is weakly special if $\alpha=0$.

Lemma 2. Every weakly special $K$ can be extended to a special $K^{*}$.

Proof. Let $K$ be weakly special. Let $\alpha_{1}, \cdots, \alpha_{r}$ be an enumeration of all $\alpha<\delta$ such that $f_{\alpha+1}^{\prime} \neq \varnothing$ in $K$. Then apply Lemma $1, r(k+1)$ times, to define the $g_{\alpha}^{\prime}(m)$, all $m \leqslant k$.

Lemma 3. Let $K$ be special, $m \in \omega, \alpha<\delta$. Then there is a special $K^{*}$ extending $K$ such that $g_{\alpha}^{\prime}(m)$ is defined in $K^{*}$.

Proof. First apply Lemma 1. Then apply Lemma 2.

Lemma 4. Let $K$ be special, $n \in \omega, \alpha<\delta$. Then there is a special $K^{*}$ extending $K$ such that $f_{\alpha+1}^{\prime}(n)$ is defined in $K^{*}$.

Proof. By Lemma 3, let $K^{\prime}$ be special, $K^{\prime}$ extending $K$, so that $g_{\alpha+1}^{\prime}(n)$ is defined in $K^{\prime}$. We may assume $g_{\alpha+1}^{\prime}(n)=\bar{x}$ in $K^{\prime}$, and $f_{\alpha+1}^{\prime}(n)$ is undefined in $K^{\prime}$. Clearly $x \in C_{\alpha+1}$. Let $y=\left\{r: g_{\alpha}^{\prime}(r)\right.$ is defined in $K^{\prime}$ and $\left.r \in a_{n}\right\}, z=\left\{s: g_{\alpha}^{\prime}(s)\right.$ is defined in $K^{\prime}$ and $\left.s \notin a_{n}\right\}$. Let $w \in \bar{x}$ be such that $y \subset w, z \cap w=\varnothing$. Let $K^{\prime \prime}=K^{\prime}+f_{\alpha+1}^{\prime}(n)=w$. Then $K^{\prime \prime}$ is weakly special. Choose a special $K^{*}$ extending $K^{\prime \prime}$ by Lemma 2 .

LEMMA 5. Let $h_{1}, \cdots, h_{r}$ be functions such that each particular one is either finite or finitely extends an element of some $C_{\mu}, \mu$ a limit $\leqslant \lambda$. Assume their domains are contained in the set of nonlimits $\gamma<\lambda$. Assume the above applies to $g$, except that $\leqslant \lambda$ is replaced by $<\lambda$. Assume $\operatorname{Rng}(g) \cap \operatorname{Rng}\left(h_{i}\right)$ $=\varnothing$ for all $i$. Let $x \in D_{\lambda}, h_{1}, \cdots, h_{r} \notin x$. Then

$$
(\exists h \in x)\left(g \subset h \& \operatorname{Rng}(h) \cap \operatorname{Rng}\left(h_{i}\right)=\varnothing, \text { for all } i\right) \text {. }
$$

Proof. By induction on limit ordinals $\lambda$. Let $\lambda=\omega$. Choose any $h^{*} \in x$. Clearly $g$ is finite, and each $h_{i}$ is either finite or eventually disagrees with $h^{*}$. There is an $n$ so large that $\operatorname{Dom}(g) \subset n$, and $\left(\operatorname{Dom}\left(h_{i}\right) \subset n\right.$ or 
$h_{i}(m) \neq h^{*}(m)$ for all $\left.m \geqslant n\right)$. Take $h(m)=h^{*}(m)$ for $m \geqslant n ; h(m) \in D_{m}$ $-\bigcup_{i} \operatorname{Rng}\left(h_{i}\right)$ for $m<n, m \notin \operatorname{Dom}(g)$; and $h(m)=g(m)$ for $m \in \operatorname{Dom}(g)$. Then $h \in x$ with the desired properties.

Suppose we have shown the lemma for all limits $\lambda^{\prime}<\lambda$, all $g, h_{1}, \cdots, h_{r}$, $x$. Now fix $g, h_{1}, \cdots, h_{r}, x$ as in the hypotheses. Choose any $h^{*} \in x$. Let $\beta<\lambda$ be so large that $\operatorname{Dom}(g) \subset \beta$, and $\left(\operatorname{Dom}\left(h_{i}\right) \subset \beta\right.$ or $h_{i}(\gamma) \neq h^{*}(\gamma)$, all nonlimits $\beta \leqslant \gamma \leqslant \lambda$ ). We can assume $\lambda>\omega$, and that $\beta$ is infinite. Let $\beta=\lambda^{\prime}+p, p \in \omega, \lambda^{\prime}$ a limit $<\lambda$. If $g \mid \lambda^{\prime} \in C_{\lambda^{\prime}}$, then take $h(\gamma)=g(\gamma)$, for $\gamma \in \operatorname{Dom}(g) ; h(\gamma)=h^{*}(\gamma)$ for $\beta \leqslant \gamma<\lambda$; and $h(\gamma) \in D_{\gamma}-\bigcup_{i} \operatorname{Rng}\left(h_{i}\right)$ for $\lambda^{\prime}<\gamma<\lambda^{\prime}+p$ and $\gamma \notin \operatorname{Dom}(g)$ (where $\gamma$ is always a nonlimit).

If $g$ | $\lambda^{\prime} \notin C_{\lambda^{\prime}}$, then $g$; $\lambda^{\prime}, h_{1}\left|\lambda^{\prime}, \cdots, h_{r}\right| \lambda^{\prime}$ satisfies the hypotheses of the lemma for $\lambda^{\prime}$. Hence by induction hypothesis, choose $g \subset g^{*} \in C_{\lambda}$, so that $\operatorname{Rng}\left(g^{*}\right) \cap \operatorname{Rng}\left(h_{i}\right)=\varnothing$, for all $i$. Finally take $h(\gamma)=g^{*}(\gamma), \gamma<\lambda^{\prime}$; $h(\gamma)=h^{*}(\gamma)$ for $\beta \leqslant \lambda$; and $h(\gamma) \in D_{\gamma}-U_{i} \operatorname{Rng}\left(h_{i}\right)$ for $\lambda^{\prime}<\gamma<\lambda^{\prime}+p$ (where $\gamma$ is always a nonlimit).

LEMMA 6. Let $h_{1}, \cdots, h_{r}$ be functions such that each particular one is either finite or finitely extends an element of some $C_{\mu}, \mu$ a limit $\leqslant \lambda$. Assume the above applies to $g$, except that $\leqslant \lambda$ is replaced by $<\lambda$. Assume $\operatorname{Rng}(g)$ $\cap \operatorname{Rng}\left(h_{i}\right)=\varnothing$, for all $i$. Let $\mu_{0}, \cdots, \mu_{s}$ be a nonrepeating sequence of limit ordinals $\leqslant \lambda, 0 \leqslant s$, and assume that $x_{0}, \cdots, x_{s}$ are such that $x_{j} \in D_{\mu_{j}}$, and $h_{i}\left\lceil\mu_{j} \notin x_{j}\right.$. Then

$$
(\exists h)\left((\forall j)\left(h \backslash \mu_{j} \in C_{\mu_{j}}\right) \& g \subset h \& \operatorname{Rng}(h) \cap \operatorname{Rng}\left(h_{i}\right)=\varnothing \text {, for all } i\right) .
$$

Proof. Apply Lemma 5 successively $s+1$ times, for $\lambda=\mu_{0}, \cdots, \mu_{s}$, after arranging $\mu_{0}, \cdots, \mu_{s}$ in increasing order. Piece together the $s+1$ functions so obtained.

Lemma 7. Let $K$ be special, $n \in \omega, \lambda$ a limit $<\delta$. Then there is a special $K^{*}$ extending $K$ such that $f_{\lambda}^{\prime}(n)$ is defined in $K^{*}$.

Proof. By Lemma 3, let $K^{\prime}$ be special and extend $K$, so that $g_{\lambda}^{\prime}(n)$ is defined in $K^{\prime}$. We may assume $g_{\lambda}^{\prime}(n)=x$ in $K^{\prime}$, and $f_{\lambda}^{\prime}(n)$ is undefined in $K^{\prime}$.

Let $k$ be as in the definition of $K^{\prime}$ being special. Then $n \leqslant k$. Define $h_{i}$, for $i \leqslant k, i \neq n$, to be the partial function on $\lambda$ given by $h_{i}(\gamma) \simeq g_{\gamma}^{\prime}(i)$, in $K^{\prime}$, for nonlimits $\gamma<\lambda$. Let $g$ be the partial function on $\lambda$ given by $g(\gamma) \simeq$ $g_{\gamma}^{\prime}(n)$, for nonlimits $\gamma<\lambda$. Let $\mu_{0}, \cdots, \mu_{s}$ list, without repetition, all limits $\mu \leqslant \lambda$ such that $(\exists i)\left(g_{\mu}^{\prime}(i)\right.$ is defined in $K^{\prime}$ and $f_{\mu}^{\prime}(i)$ is not). Choose $x_{0}$, $\cdots, x_{s}$ such that $g_{\mu_{j}}^{\prime}(i) \neq x_{j}$, for $i \neq n$, and $g_{\mu_{j}}^{\prime}(n)=x_{j}$ if defined in $K^{\prime}$, and $x_{j} \in D_{\mu_{j}}$. 
It is easily seen that $g,\left\langle h_{i}\right\rangle,\left\langle\mu_{j}\right\rangle,\left\langle x_{j}\right\rangle$ obey the hypotheses of Lemma 6 for $\lambda$. Hence we can choose $h$ such that $h i \mu_{j} \in X_{j}, g \subset h$, and $\operatorname{Rng}(h) \cap$ $\operatorname{Rng}\left(g_{i}\right)=\varnothing$, for all $i$.

Let $K^{\prime \prime}$ be the same as $K^{\prime}$ except that $f_{\mu}^{\prime}(n)=h \Vdash \mu, g_{\mu}^{\prime}(n)=[h \vdash \mu]$, $g_{\gamma}^{\prime}(n)=h(\gamma)$, and $f_{0}(n)=h(0)$, for limits $\mu \leqslant \lambda$, nonlimits $\gamma<\lambda$, in $K^{\prime \prime}$. Then $K^{\prime \prime}$ is weakly special. It should be noted that to verify condition VIII for $K^{\prime \prime}$, one uses condition IX for $K^{\prime}$.

Finally set $K^{*}$ to be any special extension by $K^{\prime \prime}$, by Lemma 2 .

Lemma 8. Let $K$ be special, $y \in D_{\alpha}, \alpha<\delta$. Then there is a special $K^{*}$ extending $K$ such that $g_{\alpha}^{\prime}(m)=y$ in $K^{*}$, for some $m \in \omega$.

Proof. Assume that $y \notin \operatorname{Rng}\left(g_{\alpha}^{\prime}\right)$ in $K$. Let $r_{1}, \cdots, r_{p}$ be a one-one enumeration of the $r$ such that $f_{\alpha+1}^{\prime}(r)$ is defined in $K$ with $y \in f_{\alpha+1}^{\prime}(r)$; let $s_{1}, \cdots, s_{q}$ be a one-one enumeration of the $s$ such that $f_{\alpha+1}^{\prime}(s)$ is defined in $K$ with $y \notin f_{\alpha+1}^{\prime}(r)$. Let $x=\bigcap_{i} a_{r_{i}} \cap\left(\bigcap_{j}\left(\omega-a_{s_{j}}\right)\right)$. Then $x$ is infinite. Choose $m \in x$ with $m \notin \operatorname{Dom}\left(g_{\alpha}^{\prime}\right)$ in $K$. Let $K^{\prime}=K+g_{\alpha}^{\prime}(m)=y$, if $\alpha \neq 0, K^{\prime}=K+g_{0}^{\prime}(m)=y+f_{0}^{\prime}(m)=y$ if $\alpha=0$. Then $K^{\prime}$ is weakly special. Extend to a special $K^{*}$ by Lemma 2 .

Theorem 2. Let $C_{\alpha}, D_{\alpha}$, for $\alpha<\delta<\omega_{1}$, be countable sets obeying $a-e$. Then there is a one-one onto $f: \omega \rightarrow \omega$ such that $C_{\alpha}=A_{\alpha}^{f}, D_{\alpha}=B_{\alpha}^{f}$, for all $\alpha<\delta$.

Proof. By Lemmas $3,4,7,8$, let $K_{0}, K_{1}, \cdots$ be a sequence of special $K_{n}$ such that (i) each $K_{n+1}$ extends $K_{n}$, (ii) for all $\alpha<\delta, m \in \omega$, there are $n, y$ such that $f_{\alpha}^{\prime}(m)=y$ in $K_{n}$, (iii) for all $\alpha<\delta, m \in \omega$, there are $n, y$ such that $g_{\alpha}^{\prime}(m)=y$ in $K_{n}$, (iv) for all $\alpha<\delta, y \in D_{\alpha}$, there are $n, m$ such that $g_{\alpha}^{\prime}(m)=y$ in $K_{n}$.

Let $f_{\alpha}(m)=y$ if and only if $(\exists n)\left(f_{\alpha}^{\prime}(m)=y\right.$ in $\left.K_{n}\right)$. Let $g_{\alpha}(m)=y$ if and only if $(\exists n)\left(g_{\alpha}^{\prime}(m)=y\right.$ in $\left.K_{n}\right)$. Take $f=f_{0}$. We claim that $C_{\alpha}=$ $A_{\alpha}^{f}, D_{\alpha}=B_{\alpha}^{f}, f_{\alpha}=f_{\alpha}^{f}, g_{\alpha}=g_{\alpha}^{f}$, all $\alpha<\delta$. It suffices to prove that $f, f_{\alpha}$, $g_{\alpha}, C_{\alpha}, D_{\alpha}$ obey conditions (1)-(9), with $A_{\alpha}, B_{\alpha}$ replaced by $C_{\alpha}, D_{\alpha}$, all $\alpha<\delta$. Conditions (1)-(5), (7)-(9) are clear. We now establish condition (6).

Suppose that $g$ has domain the nonlimits $\gamma<\lambda$, with $g(\gamma) \in D_{\gamma}, g \nmid \mu$ $\in C_{\mu}$ for limits $\mu<\lambda$, and $g(\gamma)=g_{\gamma}(m)$ for all sufficiently large $\gamma<\lambda$. Let $g^{*}=f_{\lambda}(m)$. Then $g^{*}(\gamma)=g_{\gamma}(m)$, all nonlimits $\gamma<\lambda$, and $g^{*} \in C_{\lambda}$. Hence $g \in C_{\lambda}$, since $g \sim g^{*}$.

Suppose conversely that $g \in C_{\lambda}$. Since $g_{\lambda}$ is onto, let $m, g^{*}$ be such that $f_{\lambda}(m)=g^{*}, g^{*} \sim g$. Then $g^{*} \in C_{\lambda}, g^{*}(\gamma)=g_{\gamma}(m)$, all nonlimits $\gamma<\lambda$. Hence $g$ has aomain the nonlimits $\gamma<\lambda$, with $g(\gamma) \in D_{\gamma}, g \mid \mu \in C_{\mu}$ for limits $\mu<\lambda$, and $g(\gamma)=g_{\gamma}(m)$ for all sufficiently large $\gamma<\lambda$. We are done. 
Let $|x|$ be the von Neumann cardinal of $x$. Let $\beth_{0}=\omega, \beth_{\alpha+1}=$ $\mid 2^{\beth_{\alpha}}, \beth_{\lambda}=\sup _{\alpha<\lambda} \beth_{\alpha}$.

LEMMA 9. Let $E$ be a set partitioned by $\left\{E_{n}\right\}, 0 \leqslant n$, and let $F_{n}, 0 \leqslant n$, be a collection of functions with domains $E_{n}$, whose ranges are, for fixed $n$, mutually disjoint. Assume that $\omega \leqslant\left|F_{0}\right|,\left|F_{n}\right|<\left|F_{n+1}\right|$. Then there is a set $G$ of functions with domain $E$, such that

$$
\begin{gathered}
f \in G \rightarrow f \vdash E_{n} \in F_{n}, \quad|G|=\bigcup_{n}\left|F_{n}\right|, \\
\left.(f \neq g \& f, g \in G) \rightarrow(\exists n)(\forall x)\left(f(x)=g(x) \rightarrow(\exists i<n) x \in E_{i}\right)\right),
\end{gathered}
$$

and for each $n$, there is $a G_{n} \subset G$ of power $\left|F_{n}\right|$ such that $\left(f \neq g \& f, g \in G_{n}\right)$ $\rightarrow\left(f(x)=g(x) \rightarrow(\exists i<n)\left(x \in E_{i}\right)\right)$.

Proof. Left to the reader.

THEOREM 3. There are sets $C_{\alpha}, D_{\alpha}, \alpha<\omega_{1}$, which obey (a)-(e), such that $\left|C_{\alpha}\right|=\left|D_{\alpha}\right|=\beth_{\alpha}$.

Proof. We will construct sets $C_{\alpha}, D_{\alpha}, \alpha<\omega_{1}$, obeying (a)-(e), such that $\left|C_{\alpha}\right|=\left|D_{\alpha}\right|=\beth_{\alpha}$, and for limits $\lambda^{\prime}<\lambda$, there is a subset of $C_{\lambda}$ of power $\partial_{\lambda^{\prime}}$ such that any two distinct elements disagree beyond $\lambda^{\prime}$.

Suppose the $C_{\alpha}, D_{\alpha}$ have been so defined, for $\alpha \leqslant \beta$. Define $C_{\beta+1}$ to be the closure under finite symmetric differences of some independent subset of $P\left(D_{\beta}\right)$ of power $\beth_{\beta+1}$, and $D_{\beta+1}$ to be the set of equivalence classes of elements of $C_{\beta+1}$ under finite symmetric difference.

Now suppose that the $C_{\alpha}, D_{\alpha}$ have been so defined, for all $\alpha<\lambda<\omega_{1}$. If $\lambda=\omega$, take $E=\omega, E_{n}=\{n\}$, and $F_{n}=\left\{f: \operatorname{Dom}(f)=E_{n}, \operatorname{Rng}(f) \subset D_{n}\right\}$, and choose $G$ according to Lemma 9. Set $C_{\omega}=\{g$ : $\operatorname{Dom}(g)=\omega$ and $f \sim g$ for some $f \in G\}$. Take $D_{\omega}=\left\{[g]: g \in C_{\omega}\right\}$.

Now assume that $\lambda=\mu+\omega$, some limit $\mu$. By an argument using Lemma 9, similar to the case $\lambda=\omega$, it is easy to construct $C_{\lambda}, D_{\lambda}$ of power $\beth_{\lambda}$, preserving (a)-(e), such that there is a subset $S$ of $C_{\lambda}$ of power $\beth_{\mu}$, any two distinct elements of which disagree byond $\mu$. Suppose $\lambda^{\prime}<\mu$. Then there is a subset $T$ of $C_{\mu}$ of power $\beth_{\lambda^{\prime}}$, any two distinct elements of which disagree beyond $\lambda^{\prime}$. By combining $S, T$ we get a subset of $C_{\lambda}$ of power $\beth_{\lambda^{\prime}}$, any two elements of which disagree beyond $\lambda^{\prime}$. This evidently holds for any limit $\lambda^{\prime}<\lambda$.

Finally assume $\lambda$ is a limit of limits. Let $\lambda_{0}=\omega, \lambda_{n}<\lambda_{n+1}<\lambda$, $0 \leqslant n$, and $\lim _{n} \lambda_{n}=\lambda$. Take $E$ to be the set of nonlimits $<\lambda$, and $E_{n}$ to be the set of nonlimits $\lambda_{n}<\gamma<\lambda_{n+1}$. Let $F_{0}^{\prime}$ be any infinite subset of $C_{\omega}$ any two distinct elements of which have disjoint ranges. For $0<n$, let $F_{n}^{\prime}$ be any subset of $C_{\lambda_{n}}$ of power $\beth_{\lambda_{n-1}}$ any two distinct elements of which dis- 
agree beyond $\lambda_{n-1}$. Finally, take $F_{n}$ to be the restrictions to $E_{n}$ of elements of $F_{n}^{\prime}$.

It is clear that Lemma 8 applies to $E, E_{n}$, and $F_{n}$. Let $G$ be the result of applying that lemma. Take $C_{\lambda}=\left\{g\right.$ : $\operatorname{Dom}(g)=E, G(\gamma) \in D_{\gamma}, g \vdash \mu \in C_{\mu}$, all nonlimits $\gamma<\lambda$, limits $\mu<\lambda$, and $(\exists f \in G)(f \sim g)\}, D_{\lambda}=\left\{[g]: g \in C_{\lambda}\right\}$. Then $C_{\lambda}, D_{\lambda}$ preserve (a)-(e), and have power $\beth_{\lambda}$.

Assume $\lambda^{\prime}<\lambda$. Let $\lambda_{n} \leqslant \lambda^{\prime}<\lambda_{n+1}$. Through use of the $G_{n+1} \subset G$ of Lemma 9, we see that there is a subset $S$ of $C_{\lambda}$ of power $\Sigma_{\lambda_{n+1}}$, any two distinct elements of which disagree beyond $\lambda_{n+1}$. By induction hypothesis, there is a subset $T$ of $C_{\lambda_{n+1}}$ of power $\beth_{\lambda^{\prime}}$, any two distinct elements of which disagree beyond $\lambda^{\prime}$. By combining $S, T$, we obtain a subset of $C_{\lambda}$ of power $\beth_{\lambda^{\prime}}$ any two distinct elements of which disagree beyond $\lambda^{\prime}$.

3. The models of ZF. Fix a countable transitive model $M$ of $\mathrm{ZF}, M \cap$ $O n=\lambda$. Let $x=\left\{\bar{x}_{0}, \bar{x}_{1}, \cdots\right\}$, where each $\left(x_{0}, \cdots, x_{n}\right)$ is $M$-generic. We begin by citing trivial generalizations of Theorems 2 and 3 .

For one-one onto $f: \omega \rightarrow x$, define $A_{\alpha}^{f}, B_{\alpha}^{f}, f_{\alpha}^{f}, g_{\alpha}^{f}, \alpha<\lambda$, so that $f_{0}^{f}=$ $g_{0}^{f}=f, A_{0}^{f}=B_{0}^{f}=x$, and clauses (2)-(9) of $\S 2$ hold.

Lemma 1. Suppose $C_{\alpha}, D_{\alpha}, \alpha<\lambda$, are countable sets such that $C_{0}=$ $D_{0}=x$, and $C_{\alpha}, D_{\alpha}$ obey clauses (b)-(e) of $\S 2$. Then for some one-one onto $f: \omega \rightarrow x$ we have $C_{\alpha}=A_{\alpha}^{f}, D_{\alpha}=B_{\alpha}^{f}$, for all $\alpha<\lambda$.

Let $x$ be an infinite set.

Lemma 2. There are sets $C_{\alpha}, D_{\alpha}, \alpha<\lambda, C_{0}=D_{0}=x$, obeying clauses (b) -(e) of $\S 2$, such that $\left|C_{\alpha}\right|=\left|D_{\alpha}\right| \geqslant \beth_{\alpha}$.

For sequences of sets $\left\langle S_{\alpha}\right\rangle, \alpha<\lambda$, we define $M\left[\left\langle S_{\alpha}\right\rangle\right]$ as follows. Take $M_{0}\left[\left\langle S_{\alpha}\right\rangle\right]=\varnothing . M_{\beta+1}\left[\left\langle S_{\alpha}\right\rangle\right]=\left\{y: y=T C\left(\left\{S_{\beta}\right\}\right)\right.$ or $y \in M_{\beta}$ or $y$ is firstorder definable over $\left(M_{\beta}\left[\left\langle S_{\alpha}\right\rangle\right], \in\right)$ with parameters allowed $\}, M_{\mu}\left[\left\langle S_{\alpha}\right\rangle\right]=$ $\bigcup_{\beta<\mu} M_{\beta}\left[\left\langle S_{\alpha}\right\rangle\right]$, for $\beta<\lambda, \mu$ a limit $<\lambda$. Take $M\left[\left\langle S_{\alpha}\right\rangle\right]=\bigcup_{\beta<\lambda} M_{\beta}\left[\left\langle S_{\alpha}\right\rangle\right]$.

LEMMA 3. Suppose $\left\langle S_{\alpha}\right\rangle, \alpha<\lambda$, is first-order definable over some $M(x) \vDash$ $\mathrm{ZF}$, with parameters allowed. (Hence, e.g., each $S_{\alpha} \in M(x)$.) Then $M\left[\left\langle S_{\alpha}\right\rangle\right] \vDash \mathrm{ZF}$.

PROOF. This is standard.

THEOREM. Every countable transitive model $M$ of ZF has an ordinal preserving extension satisfying $\mathrm{ZF}$ of power $\beth_{M \cap 0 n}$.

Proof. Fix $x$ to be the closure under finite symmetric differences of some $M$-generic subset of $\omega^{\omega}$. By Lemma 2, let $C_{\alpha}, D_{\alpha}, \alpha<\lambda, C_{0}=D_{0}=x$, obey clauses (b)-(e) of $\S 2$, such that $\left|C_{\alpha}\right|=\left|D_{\alpha}\right| \geqslant \beth_{\alpha}$. We will establish that $M\left[\left\langle C_{\alpha}\right\rangle\right] \vDash \mathrm{ZF}$. 
As in the proof of Theorem 1, the question of whether $M\left[\left\langle C_{\alpha}\right\rangle\right] \vDash \mathrm{ZF}$ is absolute. Thus if we can show that " $M\left[\left\langle C_{\alpha}\right\rangle\right] \vDash \mathrm{ZF}$ " holds in some Boolean extension of the universe, we will have shown that $M\left[\left\langle C_{\alpha}\right\rangle\right] \vDash \mathrm{ZF}$ is in fact true.

We show that " $M\left[\left\langle C_{\alpha}\right\rangle\right] \vDash Z F$ " holds in any Boolean extension of the universe in which $\bigcup_{\alpha<\lambda} C_{\alpha}$ becomes countable. Argue as follows in the Boolean extension. By Lemma 1, choose a one-one onto $f: \omega \rightarrow x$ such that $C_{\alpha}=A_{\alpha}^{f}$, $D_{\alpha}=B_{\alpha}^{f}$, for all $\alpha<\lambda$. By Theorem $1, M(f) \vDash \mathrm{ZF}$.

Note that $\left\langle C_{\alpha}\right\rangle$ is first-order definable over $M(f)$. Hence by Lemma 3, $M\left[\left\langle C_{\alpha}\right\rangle\right] \vDash \mathrm{ZF}$, and we are done.

In more technical terms, what we have shown is:

COROLLARY 1. Let $M$ be a countable transitive model of $\mathrm{ZF}$, and suppose $\boldsymbol{x}$ is the closure, under finite symmetric differences, of some infinite set of functions on $\omega$ that are mutually Cohen generic over $M$. Furthermore, let $C_{\alpha}, D_{\alpha}$, $\alpha \in M, C_{0}=D_{0}=x$, obey clauses (b)-(e) of $\S 2$. Then $M\left[\left\langle C_{\alpha}\right\rangle\right] \vDash \mathrm{ZF}$.

4. Hanf numbers. In Barwise [1] it is shown that the Hanf number of $L_{A}$ is $\beth_{A \cap O_{n}}$, for all countable admissible sets $A$. Is this theorem true for all admissible $A$ with countable $A \cap O n$ ?

We had answered this negatively by showing that for any countable admissible set $A$, there is an ordinal preserving admissible extension $B$ such that the Hanf number of $L_{B}$ is $>\beth_{A}$ non. Furthermore, $B$ can be taken to be the least admissible set $B \supset A$ with $x \in B$, for some $x \subset \omega^{\omega}$ depending on $A$. The proof had no connection with the methods introduced in this paper. The proof does not construct $B \vDash \mathrm{ZF}$.

Leo Harrington has shown, by an application of the methods introduced here, that every countable transitive model $M \vDash \mathrm{ZF}$ has an ordinal preserving extension $N \vDash Z$ ZF such that the Hanf number $L_{N}$ is greater than $Z_{c^{+}}$. (Also, if $\vDash \mathrm{ZF}$ is replaced by admissibility.) This is an easy consequence of the following.

COROLlaRY 2. Let $M$ be a countable transitive model of $\mathrm{ZF}$, and suppose $x$ is the closure, under finite symmetric differences, of some infinite set of functions on $\omega$ that are mutually Cohen generic over $M$. Then $M(P(x)) \vDash \mathrm{ZF}$.

ProOF. The proof is the same as that of Corollary 1, except that the combinatorial lemma of $\S 2$ is replaced by: the closures of any two countable atomless Boolean algebras of subsets of an infinite set, under finite symmetric difference, are isomorphic.

COROllary 3. Every countable transitive model $M$ of ZF has an ordinal preserving extension $N$ of $\mathrm{ZF}$ such that the Hanf number of $L_{N}$ is greater than ${ }_{c}+\cdot$ 
Proof. By Corollary 2, we may choose $x$ of power $c$ so that $M(P(x))=$ $N \vDash \mathrm{ZF}$. There is a sentence $\phi$ in $L_{N}$ whose models have the following apparatus: (i) a model $(A, R)$ of the axioms of admissibility, (ii) for each $a \in A$ such that $(A, R) \vDash$ " $a$ is an ordinal," an isomorphism between $(A \vdash a$, $R(a)$ and some linear ordering $l_{a}$ on a subset of $x$, (iii) $l_{a}$ is well ordered with respect to all subsets of its field that are in $N$.

Since $P(x) \in N$, clearly (iii) requires that each $l_{a}$ is a well ordering. Hence $(A, R)$ must be well founded, and of height at most $c^{+}$. Clearly $c^{+}$is possible by taking $\left(V\left(c^{+}\right), \in\right)$. The maximum cardinality of models of $\phi$ is therefore $\beth_{c^{+}}$, and so the Hanf number of $L_{N}$ is greater than $\beth_{c^{+}}$.

Corollaries 2 and 3 can be strengthened by combining "the closures of any two countable atomless Boolean algebras of subsets of an infinite set under finite symmetric differences are isomorphic" with the transfinite constructions of $\S 2$.

Replace conditions (a)-(e) of $\S 2$ by the following conditions on $x, C_{\alpha}$, $D_{\alpha}, E_{\alpha}, F_{\alpha}, \alpha<\omega_{1}$ :

(a') $C_{0}=x, D_{0} \cup E_{0}=x, D_{0} \cap E_{0}=\varnothing, D_{0}, E_{0}$ infinite, $F_{0}$ is the closure under finite symmetric differences of some infinite atomless Boolean algebra of subsets of $E_{0}$.

(b') Same as b.

(c') $D_{\alpha+1} \cap E_{\alpha+1}=\varnothing, D_{\alpha+1} \cup E_{\alpha+1}=\left\{\bar{x}: x \in C_{\alpha+1}\right\}, D_{\alpha+1}, E_{\alpha+1}$ are infinite, $F_{\alpha+1}$ is the closure of some infinite atomless Boolean algebra of subsets of $E_{\alpha+1}$ under finite symmetric differences.

(d') Same as d.

(e') $D_{\lambda} \cap E_{\lambda}=\varnothing, D_{\lambda} \cup E_{\lambda}=\left\{[f]: f \in C_{\lambda}\right\}, D_{\lambda}, E_{\lambda}$ are infinite, $F_{\lambda}$ is the closure of some infinite atomless Boolean algebra of subsets of $E_{\lambda}$ under finite symmetric differences.

By suitably modifying (1)-(9), and I-IX of $\S 2$ and imitating the proof of Corollary 1 , we obtain the following

COROLLARY 4. Let $M$ be a countable transitive model of $\mathrm{ZF}$, and suppose $x$ is the closure, under finite symmetric difference, of some infinite set of functions on $\omega$ that are mutually Cohen generic over $M$. Furthermore, let $x$,

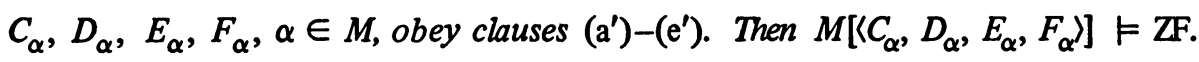

An obvious modification of the proof of Theorem 3 yields the following.

CoRollary 5. Let $M$ be a countable transitive model of ZF. Then there is an ordinal preserving extension $N$ satisfying ZF such that, for each $\alpha \in N$, there is an $x \in N$ with $|x|=\beth_{\alpha}$ and $P(x) \in N$.

Proof. Arrange $\left|C_{\alpha}\right|=\left|D_{\alpha}\right|=\left|E_{\alpha}\right|=\beth_{\alpha}$, and $F_{\alpha}=P\left(E_{\alpha}\right)$.

COROLLARY 6. Every countable transitive model $M$ of $\mathrm{ZF}$ has an ordinal 
preserving extension $N$ satisfying ZF such that the Hanf number $L_{N}$ is ${ }_{\mathrm{I}_{M \cap O n}}$.

Proof. This is obtained from Corollary 5 in the same way that Corollary 3 is obtained from Corollary 2. The Hanf number of $N$ cannot exceed $\beth_{\beth_{M} \cap O_{n}}$ since $L_{N} \subset U_{k<z_{M \cap O n}} L_{k \omega}$, and the latter has Hanf number $I_{\beth_{M \cap O n}}$, by Chang [2] and Morley [3].

We conclude the paper by briefly considering possibly nonstandard models of $\mathrm{ZF}$, answering a question posed to us by Sy Friedman.

COROLlaRY 7. For each $\beta<\omega_{1}$ there are models of ZF of any infinite power, which have countably many ordinals and whose standard ordinal is at least $\beta$.

Proof. This follows immediately from the fact that there are such models of each power $<\beth_{\omega_{1}}$, since the Hanf number of $L_{\omega_{1} \omega}$ is $\beth_{\omega_{1}}$.

\section{REFERENCES}

1. J. Barwise, Infinitary logic and admissible sets, Doctoral Dissertation, Stanford University, Stanford, Calif., 1967.

2. C. C. Chang, Some remarks on the model theory of infinitary languages, The Syntax and Semantics of Infinitary Languages, Lecture Notes in Math., vol. 72, Springer-Verlag, Berlin, 1968, p. 47.

3. M. Morley, Omitting classes of elements, Theory of Models (Proc. 1963 Internat. Sympos. Berkeley), North-Holland, Amsterdam, 1965, pp. 265-273. MR 34 \#1189.

DEPARTMENT OF MATHEMATICS, STATE UNIVERSITY OF NEW YORK AT BUFFALO, AMHERST, NEW YORK 14226 